\title{
A study on the association of lipid profiles with HbA1C levels and drug prescription in type 2 diabetes mellitus
}

\author{
M. Rakshna, D. Sheela* and Porchelvan Swaminathan \\ Department of Pharmacology, Saveetha Medical College and Hospital, SIMATS, \\ Chennai, 602105, India.
}

\begin{abstract}
Diabetes mellitus has become a pandemic and a predominant health problem due to its rapid and increased prevalence in developed and developing countries. Diabetes is a metabolic disorder characterised by hyperglycaemia. The increase in glucose level is due to inappropriate secretion or action of insulin. Diabetes has produced a significant impact on morbidity and mortality rate. The various complications produced due to chronic diabetes include retinopathy, nephropathy and neuropathy. The major health problem includes occurrence of macrovascular complications like coronary artery diseases. Long-standing poor glycemic control results in hyperlipidemia, hypertension and obesity. This prospective, observational and cross-sectional study was undertaken as a short time project for a period of 3 months (December 2018-February 2019 by a second year MBBS student. The study was conducted in the Outpatient Department of Medicine by the Department of Pharmacology. The study was initiated after obtaining approval from the human ethics committee and from the patients by voluntary consent. Around 150 patients who were confirmed with type 2 diabetes were selected to be part of the study. The patients were grouped according to their HbA1c levels (7-8\%, 8-10\% and above $10 \%$ ) and each group had 50 patients. Total cholesterol (TC), triglycerides (TGL), high density lipoprotein cholesterol (HDL), low density
\end{abstract}

*Corresponding author: sheelapharm15@yahoo.in lipoprotein cholesterol (LDL), very low density lipoprotein cholesterol (VLDL), fasting blood glucose and postprandial blood glucose were measured in these patients. The present study confirmed elevation in lipid parameters with rise of HbA1c levels. Thus, the study clearly identified the need for early diagnosis of lipid levels in type 2 diabetes in order to prescribe suitable drugs by which further cardiovascular complications can be reduced.

KEYWORDS: dyslipidemia, diabetes mellitus, hypertension, postprandial blood glucose.

\section{INTRODUCTION}

Diabetes mellitus has become a pandemic and a predominant health problem due to its rapid and increased prevalence in developed and developing countries. The universal prevalence of diabetes in the age group of 27-79 years is estimated to be around 640 million in 2040. Diabetes is a metabolic disorder characterised by hyperglycaemia [1]. In the Indian population, about 5.2 crores are expected to be affected with diabetes by 2025 [2]. The increase in glucose levels is due to inappropriate secretion or action of insulin. Diabetes has produced a significant impact on morbidity and mortality rate. The various complications produced due to chronic diabetes include retinopathy, nephropathy and neuropathy. The major health problem includes the occurrence of macrovascular complications like coronary artery diseases $[3,4]$. Long-standing poor glycemic control results in hyperlipidemia, hypertension and obesity. Diabetes 
with dyslipidemia shows a linear relationship to cardiovascular diseases in comparison with nondiabetic patients [5]. Dyslipidemia is also now called as diabetes lipidus [6]. Diabetic dyslipidemia includes a classic pattern of an abnormally raised level of triglycerides, low density proteins and marked fall in high density proteins. The abnormal lipid levels in diabetes mellitus end up with insulin resistance [7]. Therefore, serious and necessary steps should be taken to prevent complications due to diabetes. The American Diabetes Association (ADA) has recommended HbA1c as a better indicator for diagnosis of diabetes which can reflect the cumulative glycemic history of proceeding two or three months. The raised HbA1c is considered as an independent risk factor for coronary artery diseases and stroke in diabetic patients [8]. The American Diabetes Association has correlated $\mathrm{HbA1c}$ and cardiovascular disease complication in which $1 \%$ increase in HbA1c level will result in $25 \%$ increase in diabetesrelated mortality, 35\% increase in microvascular complications and $18 \%$ increase in myocardial infarction [9]. Patients with uncontrolled glycemia are more prone to dyslipidemic progression. An increase in total cholesterol above $180 \mathrm{mg}$ and a decrease in HDL level less than $35 \mathrm{mg} / \mathrm{dl}$ results in 8 times increase in coronary artery disease (CAD) in comparison with HDL above $65 \mathrm{mg} / \mathrm{dl}$. A trial on lipid research concludes that $1 \%$ fall in TC results in $2 \%$ reduction in CAD. Another study showed that $12 \%$ increase in HDL and $11 \%$ decrease in LDL will result in 34\% reduction in CAD [10]. In developing countries where most of the people live below the poverty line, the frequent collection of blood for diagnosis of diabetes and dyslipidemia is becoming tedious and uneconomical. The glycated haemoglobin estimation can be considered as a productive way for the early diagnosis for both diabetes and dyslipidemia. In turn, this helps in reducing other cardiovascular complications [11]. The study was carried out to investigate the relationship between increased HbA1c levels with the lipid levels and the treatment provided in type 2 diabetes mellitus patients.

\section{PATIENTS AND METHODS}

This prospective, observational and cross-sectional study was undertaken as a short time project for a period of 3 months (December 2018-February 2019) by a second-year MBBS student. The study was conducted in the Outpatient Department of Medicine by the Department of Pharmacology. The study was initiated after obtaining approval from the human ethics committee and from the patients by voluntary consent. Around 150 patients who were confirmed with type 2 diabetes were selected to be part of the study. The patients were grouped according to their HbA1c levels (7-8\%, $8-10 \%$ and above $10 \%$ ) and each group had 50 patients. Total cholesterol (TC), triglycerides (TGL), high density lipoprotein cholesterol (HDL), low density lipoprotein cholesterol (LDL), very low density lipoprotein cholesterol (VLDL), fasting blood glucose and postprandial blood glucose were measured in these patients.

National Cholesterol Education Program (NCEP) Adult Treatment III (ATPIII) 2018 guideline was used as a reference for the lipid levels. According to NCEP ATPIII guidelines, hypercholesterolemia is defined as TC $>200 \mathrm{mg} / \mathrm{dl}$, LDL $>100 \mathrm{mg} / \mathrm{dl}$, TGL $>150 \mathrm{mg} / \mathrm{dl}$ and HDL $<40 \mathrm{mg} / \mathrm{dl}$. Dyslipidemia is defined as the presence of one or more abnormal serum lipid concentration. Diabetes was defined according to the American Diabetes Association (ADA) criteria. The data was evaluated using the SPSS Statistical package, version 17.0. Independent t-test was used to compare the means of different parameters. Analysis of variance, Mann-Whitney Test, and Kruskal-Wallis Test were used for the statistical calculation. HbA1c was given as a percentage of total haemoglobin and values of others were given in $\mathrm{mg} / \mathrm{dl}$. The result was considered to be significant when $\mathrm{P}$ values were less than 0.05 .

\section{Inclusion criteria}

Confirmed type 2 diabetes mellitus patients who regularly visit the hospital with HbA1c values below $15 \%$ and aged above 18 years were included in the study. After obtaining verbal consent from the patients, the prescription and other lab details were collected for the study and the results were maintained confidential.

\section{Exclusion criteria}

Type 2 diabetic patients with chronic kidney disease, diabetic foot, with recent surgery and with major complications like retinopathy and 
HbA1c levels above 15 were excluded from the study.

\section{RESULTS}

Among 150 patients, 60 were females and 90 were males and they were grouped according to the different HbA1c levels. The mean age group in male patients was $50.32 \pm 10.90$ and female patients was $51.73 \pm 13.10$ years. The $\mathrm{HbA1c}$ levels in females were $8.98 \pm 2.45$ and in males were $9.80 \pm 2.18$. The fasting blood glucose levels in females were $176.72 \pm 64.48$ and males were $208.83 \pm 63.89$. The postprandial blood glucose levels in females were $259.19 \pm 96.86$ and males were $292.65 \pm 90.34$.

\section{DISCUSSION}

In diabetes mellitus, increased hyperglycaemia is responsible for cardiovascular complications. The mortality rate in these patients is more than $70 \%$ compared to the normal individual. Metabolic abnormality like dyslipidemia is associated with type 2 diabetes mellitus which further increase the cardiovascular complications [12]. The lipid profile and diabetes are the important prognostic factors for these metabolic disturbances. Type 2 diabetes mellitus individuals with dyslipidemia normally have the characteristic feature of increased triglycerides levels and decreased HDL levels. Elevated levels of non-HDL and the presence of denser LDL are responsible for the cause of atherogenicity in diabetes mellitus patients [4].

The present study showed increased levels of TG, LDL and VLDL when compared with the normal lipid profile in these individuals. The LDL, VLDL and TGL levels in HbA1c 8-10\% group was found to be $121 \%, 123 \%, 130 \%$ and in $\mathrm{HbA} 1 \mathrm{c}$ above $10 \%$ group, it was found to be $167 \%$, $130 \%, 150 \%$ whereas in the category of HbA1c levels $7-8 \%$, the lipid parameters were in normal range (Figure 1). According to recent guidelines of the American College of Physician, the HbA1c levels can be $7-8 \%$ in diabetic individuals [13]. A previous study conducted by Masram et al. found that dyslipidemia was more predominant in type 2 diabetes than type 1 diabetes which was confirmed by an increased level of TC and decreased level of HDL whereas in the current study there were no significant changes in these parameters [14]. In an earlier study, the lipid profile showed a statistically significant increase whereas in our study the TG, LDL and VLDL showed increased level but they were not statistically significant among the groups (Table 1). The result was similar in the study conducted by Biradar et al. [15]. In another study,

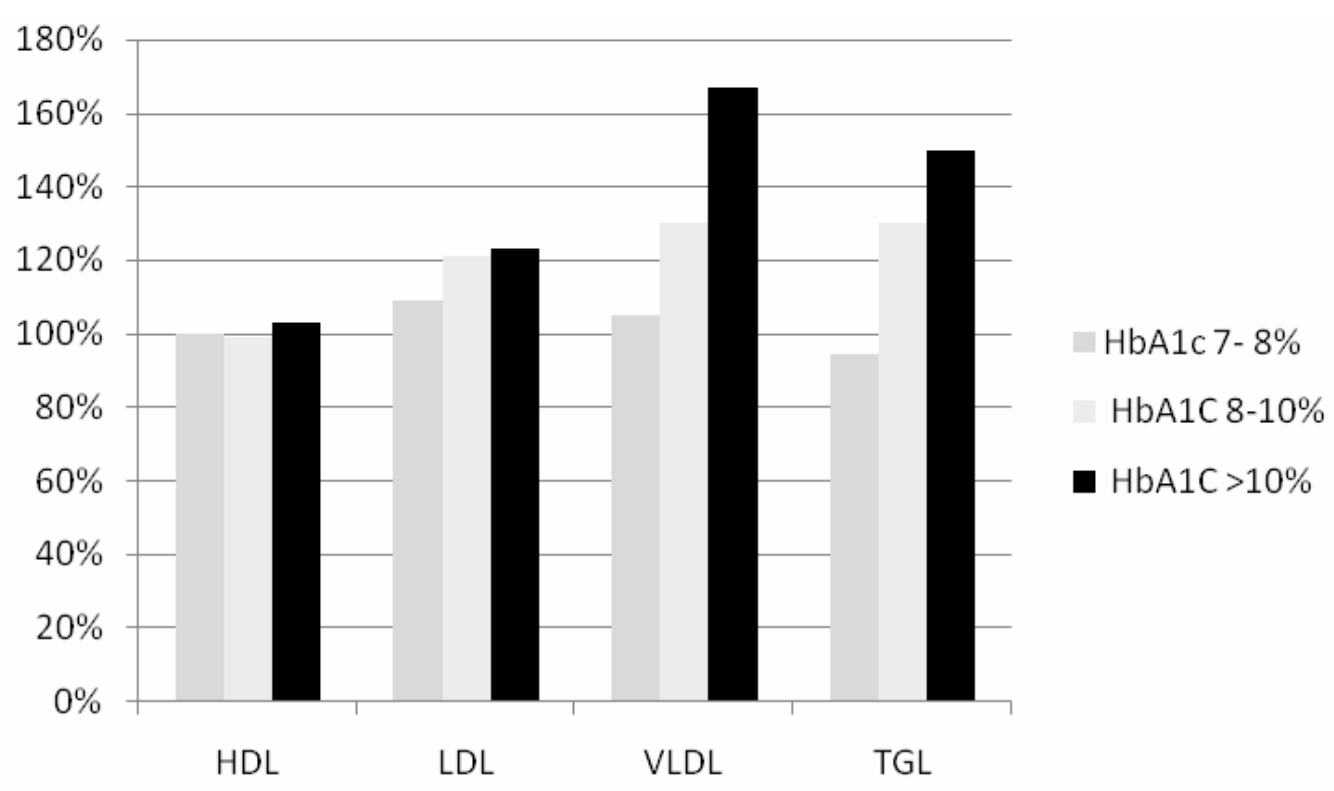

Figure 1. Lipid levels in type 2 diabetes categorized according to their HbA1c levels. 
Table 1. Categorization of type 2 diabetic mellitus patient's lipid levels according to HbA1c levels.

\begin{tabular}{|c|c|c|c|c|}
\hline \multirow[b]{2}{*}{ Parameter } & \multicolumn{3}{|c|}{ Glycated hemoglobin (HbA1C) } & \multirow[b]{2}{*}{ Significance } \\
\hline & $\begin{array}{c}7.0-8.0(\mathrm{~N}=50) \\
\text { Mean } \pm \mathrm{SD}\end{array}$ & $\begin{array}{c}\text { 8-10 }(\mathrm{N}=50) \\
\text { Mean } \pm \text { SD }\end{array}$ & $\begin{array}{l}\text { Above } 10(N=50) \\
\text { Mean } \pm \text { SD }\end{array}$ & \\
\hline TC & $175.40 \pm 31.40$ & $199.85 \pm 45$ & $202.50 \pm 43$ & 0.074 \\
\hline TGL & $142.10 \pm 30.12$ & $195.45 \pm 80.56$ & $224.75 \pm 159.08$ & 0.120 \\
\hline HDL-C & $40.05 \pm 21.31$ & $39.55 \pm 5.92$ & $41.45 \pm 21.33$ & 0.334 \\
\hline LDL-C & $109.10 \pm 29.66$ & $121.36 \pm 42.32$ & $123.71 \pm 32.32$ & 0.377 \\
\hline VLDL & $31.75 \pm 14.77$ & $39.09 \pm 16.11$ & $50.24 \pm 36.46$ & 0.224 \\
\hline FBS mg/DL & $118.45 \pm 33.02$ & $222.30 \pm 38.44$ & $243.25 \pm 36.97$ & $0.000 *$ \\
\hline PPBS mg/DL & $179.75 \pm 54.97$ & $318.90 \pm 62.13$ & $335.80 \pm 71.17$ & $0.000 *$ \\
\hline
\end{tabular}

*Statistically significant.

taking HbA1c of $9 \%$ as cut off value resulted in similar results with respect to TG, LDL levels [10]. A significant correlation was found in fasting and the postprandial blood glucose levels with HbA1c levels [16]. Various studies conducted earlier highlighted the relationship between hypoglycaemia and HbA1c levels [17, 18]. The present study also confirms this relationship via the glucose and HbA1c levels in these individuals. A drastic increase in glucose levels was seen with an elevation of HbA1c levels. The present study also failed to show a significant difference in lipid parameters and the fasting and postprandial glucose levels among male and female patients [10] (Table 2). The present study shows a significant correlation of HbA1c with triglycerides only whereas in earlier studies all the lipid parameters were significantly correlated [19]. The fasting blood glucose showed a negative correlation with HDL-C [14] (Table 3).

According to the American Diabetes Association (ADA), the first and foremost treatment recommended in these patients is Medical Nutritional Therapy. This includes a reduction in saturated fat, trans fat and cholesterol intake. In addition, the American College of Cardiology guidelines suggest weight reduction and exercise. The pharmacological treatment in these individuals includes the use of statins, niacin, fibrate and bile acid sequestrants which can be given as monotherapy or in combinations to reduce the elevated cholesterol levels. The clinical studies conducted for lowering cholesterol levels in diabetic patients with the use of HMG Co-A reductase or statins showed a significant reduction in coronary heart disease and mortality rate with high LDL levels. Pravastatin, another statin was also used in moderate level of LDL. A meta-analysis of 14 randomised control trials stipulated the use of statins in which a proportional decrease in mortality rate was demonstrated. The most common adverse effects of statins are headache, non-specific muscle and joint pain, nausea, diarrhoea, constipation and abdominal pain. Therefore, the patients using statins who experience unexplained muscle weakness, pain or fever are advised to consult the physician immediately [20, 21, 22].

Niacin is used to treat dyslipidemia in type 2 diabetes patients for more than 50 years. It has been used for the effective increase in HDL levels whereas an increase in dose may result in hyperglycemia. The other adverse effects seen are flushing, itching, nausea, gastrointestinal upset, hypotension and tachycardia. The flushing produced by niacin can be overcome by the use of dopamine (D2) antagonist laropiprant. Ezetimibe, a selective cholesterol absorption inhibitor and bile acid sequestrant coleselvam, are the other drugs that are used to reduce the cholesterol levels. These can be used either as monotherapy or in combination with statins to reduce the adverse effect of individual drugs [3]. The drugs used for reducing increased glucose levels are metformin and pioglitazone. Metformin used for hyperglycemia has an additional effect of reducing 
Table 2. Lipid parameters in male and female type 2 diabetic patients.

\begin{tabular}{|l|c|c|c|}
\hline Parameter & $\begin{array}{c}\text { Females (n= 60) } \\
\text { Mean } \pm \text { SD }\end{array}$ & $\begin{array}{c}\text { Males (n = 90) } \\
\text { Mean } \pm \text { SD }\end{array}$ & Significance \\
\hline HbA1c & $8.98 \pm 2.45$ & $9.80 \pm 2.18$ & 0.175 \\
\hline TC & $184.96 \pm 36.16$ & $198.41 \pm 45.04$ & 0.218 \\
\hline TGL & $185.58 \pm 136.11$ & $188.85 \pm 83.17$ & 0.909 \\
\hline HDL-C & $39.46 \pm 7.74$ & $41.03 \pm 16.52$ & 0.656 \\
\hline LDL-C & $109.90 \pm 31.06$ & $124.28 \pm 37.29$ & 0.118 \\
\hline VLDL & $39.82 \pm 28.84$ & $40.77 \pm 22.69$ & 0.887 \\
\hline FBS mg/DL & $176.72 \pm 64.48$ & $208.83 \pm 63.89$ & 0.063 \\
\hline PPBS mg/DL & $259.19 \pm 96.86$ & $292.65 \pm 90.34$ & 0.174 \\
\hline
\end{tabular}

Table 3. Correlation between glycemic markers and lipid levels in type 2 diabetes mellitus patients.

\begin{tabular}{|c|c|c|c|c|c|}
\hline & TGL & T.CHOL & LDL & HDL & VLDL \\
\hline \multirow{2}{*}{ HbA1C } & R 0.343 & 0.229 & 0.118 & 0.013 & 0.304 \\
\cline { 2 - 6 } & $\mathrm{p} 0.007^{*}$ & 0.078 & 0.371 & 0.919 & 0.018 \\
\hline \multirow{2}{*}{ FBS } & $\mathrm{r} 0.292$ & 0.281 & 0.163 & -0.005 & 0.209 \\
\cline { 2 - 6 } & $\mathrm{p} 0.024^{*}$ & $0.030^{*}$ & 0.214 & 0.968 & 0.110 \\
\hline \multirow{2}{*}{ PPBS } & $\mathrm{r} 0.142$ & 0.195 & 0.201 & 0.048 & 0.148 \\
\cline { 2 - 6 } & $\mathrm{p} 0.281$ & 0.136 & 0.124 & 0.717 & 0.259 \\
\hline
\end{tabular}

*Correlation is significant at $\mathrm{P}>0.05$.

LDL, TC, TGs and increasing HDL whereas pioglitazone reduces TG and increases HDL $[23,24]$.

In the present study, the drugs used for hyperglycemia were glimipride, metformin, sitagliptin, vidagliptin, tenegliptin, glibenclamide, glipizide and insulin. The drugs used for dyslipidemia were atorvastatin, rosuvastatin, and pitavastatin. It has been found that the use of these drugs produces beneficial effect in these diabetic people by controlling their lipid profile and glucose levels.

The present study had some limitations and it was the sample size and research period. Since the study was a short project for second year medical students, the sample size was smaller and the period of research was only 3 months. The subjects included in the study were already identified as type 2 diabetes mellitus and dyslipidemic patients, and there were no newly diagnosed patients.

\section{CONCLUSION}

Dyslipidemia in type 2 diabetes mellitus patients often results due to the presence of low HDL-C, high LDL-C and TGs. Currently, HbA1c is considered to be an effective biomarker in diagnosing both diabetes and dyslipidemia. The present study was carried with the objective of identifying the correlation between HbA1c levels and lipid profile levels. The study failed to find significant correlation with lipid profiles which was present in the earlier studies. But, the lipid parameters showed an increase with the rise in HbA1c levels. Therefore, a study devoid of the above-mentioned limitations can help in rendering 
accurate results which could be in harmony with previous studies. Thus, the present study clearly identified the need for early diagnosis of lipid levels in type 2 diabetes patient in order to prescribe suitable drugs and to reduce further cardiovascular complications.

\section{CONFLICT OF INTEREST STATEMENT}

The authors declare that there is no conflict of interest.

\section{REFERENCES}

1. Hussain, A., Ali, I., Ijaz, M. and Rahim, A. 2017, Therapeutic Advances in Endocrinology and Metabolism, 4, 51.

2. Singh, G. and Kumar, A. 2011, Journal of Exercise Science and Physiotherapy, 7, 99.

3. Kolhar, U. and Priyanka, P. 2017, Int. J. Adv. Med., 4, 1513.

4. Vijayaraghavan, K. 2010, Lipids in Health and Disease, 9, 144.

5. Ozder, A. 2014, Lipids in Health and Disease, 13, 183.

6. Mullugeta, Y., Chawla, R., Kebede, T. and Worku, Y. 2012, Indian Journal of Clinical Biochemistry, 27, 363.

7. Alam, R., Verma, M. K. and Verma, P. 2015, International Journal of Life-Sciences Scientific Research, 1, 62.

8. Lodha, R., Lal, R. and Biyani, S. 2016, Scholar Journal of Applied Medical Sciences, 4, 1600.

9. Pinnelli, V. B. 2016, International Journal of Research in Medical Sciences, 4, 4524.

10. Prabhavathi, K., Kunikullaya, K. and Goturu, J. 2014, Journal of Clinical and Diagnostic Research, 8, 20.

11. Baranwal, J. K., Maskey, R., Majhi, S., Lamsal, M. and Baral, N. 2015, Health Renaissance, 13, 16.
12. Laakso, M. 2010, Diabetes Care, 33, 442.

13. Qaseem, A, Wilt, T. J., Kansagara, D., Horwitch, C., Barry, M. J. and Forciea, M. A. 2018, Annals of Internal Medicine, 168, 569.

14. Masram, S. W., Bimanpalli, M. V. and Ghangle, S. 2012, Indian Medical Gazette, 145, 257.

15. Biradar, S. B., Desai, A. S., Kashinakunti, S. V., Rangappa, M., Kallaganada, G. S. and Devaranavadagi, B. 2018, International Journal of Advances in Medicine, 5, 1.

16. Mandal, G. K. and Jha, P. K. 2017, International Journal of Contemporary Medical Research, 4, 1408.

17. Sherwani, S. I., Khan, H. A., Ekhzaimy, A., Masood, A. and Sakharkar, M. K. 2016, Biomarker Insights, 11, 38440.

18. World Health Organization. 2011, Use of glycated haemoglobin (HbA1c) in diagnosis of diabetes mellitus: abbreviated report of a WHO consultation. World Health Organization. http://www.who.int/iris/handle/10665/70523

19. Singh, G. and Kumar, A. 2011, Journal of Exercise Science and Physiotherapy, 7, 99.

20. Bantle, J. P., Wylie-Rosett, J., Albright, A. L., Apovian, C. M., Clark, N. G., Franz, M. J., Hoogwerf, B. J., Lichtenstein, A. H., Mayer-Davis, E., Mooradian, A. D. and Wheeler, M. L. 2008, Diabetes Care, 31, 61.

21. American Diabetes Association. 2003, Diabetes Care, 26, 83.

22. Naqvi, S., Naveed, S., Ali, Z., Ahmad, S. M., Khan, R. A., Raj, H., Shariff, S., Rupareliya, C., Zahra, F. and Khan, S. 2017, Cureus, 9, 1347.

23. Kashi, Z., Mahrooz, A., Kianmehr, A. and Alizadeh, A. 2016, PLoS One, 11, e0151543.

24. Sen, S., Sinha, S. and Gupta, K. K. 2013, Int. J. Basic Clin. Pharmacol., 2, 257. 